\title{
ON THE TRUNCATED SMALL FUNCTION THEOREM IN NEVANLINNA THEORY
}

\author{
KATSUTOSHI YAMANOI
}

\section{INTRODUCTION}

The main purpose of this paper is to prove the theorem below, which improves upon a result of $[\mathrm{Y}]$ by eliminating the hypothesis that $b: B \rightarrow M$ is Zariski dense and by establishing the independence of the constant $C$ from $g: Y \rightarrow X$. As we shall see later, this theorem unifies two main results of $[Y]$ : the truncated $q$-small function theorem, and the height inequality for curves over function fields conjectured by P. Vojta [V2]. To state our theorem explicitly, we need one definition.

Definition of the exceptional locus . Let $X$ and $M$ be smooth complex projective varieties, and let $p: X \rightarrow M$ be a surjective morphism where the relative dimension of $X$ over $M$ is equal to one. Let $D \subset X$ be a reduced divisor. The exceptional locus $Z(X, M, p, D)$ is a proper Zariski closed subset of $M$ defined as follows: Let $\mathcal{U}$ be the set of all Zariski open subsets $U$ on $M$ such that (1) the restriction $\left.p\right|_{U}: p^{-1}(U) \rightarrow U$ is a smooth morphism, and (2) the restriction of the divisor $D$ on the fiber $p^{-1}(x)$ is a reduced divisor for every point $x \in U$. Put

$$
Z=Z(X, M, p, D)=M \backslash \bigcup_{U \in \mathcal{U}} U
$$

Then, since $X$ is smooth and $D$ is reduced, this $Z$ is a proper Zariski closed subset of $M$.

Now we state our main result. (The notation in the theorem concerning Nevanlinna theory will be given in the body of this paper.)

Theorem . Let $X, M, p$ and $D$ be the same as above. We denote by $K_{X / M}=K_{X}-p^{*} K_{M}$ the relative canonical line bundle. Let $L$ and $E$ be ample line bundles on $X$ and $M$, respectively, and let $\varepsilon>0$. Then there exists a positive constant $C=C(X, M, p, D, L, E, \varepsilon)$ with the following property: Let $Y$ and $B$ be Riemann surfaces with proper, surjective holomorphic maps $\pi_{Y}: Y \rightarrow \mathbb{C}$ and $\pi_{B}: B \rightarrow \mathbb{C}$. Assume that $\pi_{Y}$ factors through $\pi_{B}$, i.e., there exists a proper, surjective holomorphic map $\pi: Y \rightarrow B$ such that $\pi_{Y}=\pi_{B} \circ \pi$. Consider the following commutative diagram of holomorphic maps where $g$ is non-constant.

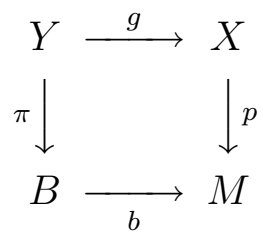

Assume that $b(B) \not \subset Z(X, M, p, D)$ and $g(Y) \not \subset \operatorname{supp} D$. Then we have

$$
\begin{aligned}
T\left(r, g, K_{X / M}(D)\right) \leq \bar{N}(r, g & , D)+N_{\operatorname{ram} \pi_{Y}}(r) \\
& +\varepsilon T(r, g, L)+C\left(T(r, b, E)+N_{\operatorname{ram} \pi_{B}}(r)\right)+O(1) \|
\end{aligned}
$$


Here the symbol "I|" means that the stated estimate holds for $r>0$ outside some exceptional interval with finite Lebesgue measure. Our theorem has a non-trivial conclusion even for the case that $g$ and $b$ are not transendental. Namely, we have the following

Corollary 1. Let $X, M, p, D, L$ and $E$ be the same as the theorem, and let $\varepsilon>0$. Then there is a positive constant $C=C(X, M, p, D, L, E, \varepsilon)$ with the following property: Let $Y$ and $B$ be compact Riemann surfaces with a proper, surjective holomorphic map $\pi: Y \rightarrow B$. Consider the following commutative diagram of holomorphic maps where $g$ is non-constant.

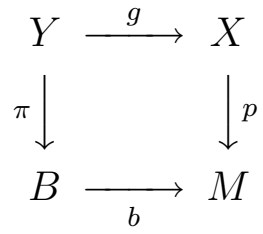

Assume that $b(B) \not \subset Z(X, M, p, D)$ and $g(Y) \not \subset \operatorname{supp} D$. Then we have

$$
\begin{aligned}
\operatorname{deg} g^{*} K_{X / M}(D) \leq \bar{n}(g, D, Y)+ & 2 \operatorname{genus}(Y)+\varepsilon \operatorname{deg} g^{*} L \\
& +C(\operatorname{deg} \pi)\left(\operatorname{deg} b^{*} E+\operatorname{genus}(B)+1\right) .
\end{aligned}
$$

Here we denote by $\bar{n}(g, D, Y)$ the cardinal number of the finite set $\{z \in Y ; g(z) \in D\}$. If we apply this corollary to the case that $M$ is a compact Riemann surface, $B=M$ and $b=\mathrm{id}_{M}$, we immediately get the height inequality for curves over function fields, which is a special case of a conjecture proposed by Vojta [V2]. See also [Y, Section 9].

The proof of the theorem basically follows the procedure of the proof of [Y, Corollary 3], where the estimate (1.0.1) is proved under the additional condition that $b(B)$ is Zariski dense in $M$. Also the independence of the constant $C$ from $Y, B, \pi, g$ and $b$ is our new observation.

The plan of this paper is the following. In section 2, we introduce notation and preliminary results. In section 3, we prove two lemmas which will be needed for the theorem. In section 4, we prove the theorem. In section 5, we derive Corollary 1 from the theorem. Our theorem implies the truncated $q$-small function theorem very simply. We shall explain this in section 6 .

Correction to the literature $[\mathrm{Y}]$. On the last sentence of page 226, the condition "when $r \notin E$ for some exceptional set $E \subset \mathbf{R}_{>0}$ "

should be

$$
\text { "when } r>2 \text { and } r \notin E \text { for some exceptional set } E \subset \mathbf{R}_{>2} \text { ". }
$$

While we have estimated the exceptional interval $E$ by $\int_{E} d \log \log r<\infty$ in [Y], we shall estimate $E$ by $\int_{E} d r<\infty$ in this paper.

\section{Notation And Preliminaries}

2.1. Notation. Let $Y$ be a Riemann surface, and let $\Omega \subset Y$ be a relatively compact open subset. Let $X$ be a smooth projective variety, and let $D$ be an effective divisor. Given a holomorphic map $g: Y \rightarrow X$ such that $g(Y) \not \subset$ supp $D$, we put

$$
\bar{n}(g, D, \Omega)=\sum_{x \in \Omega} \min \left\{1, \operatorname{ord}_{x} g^{*} D\right\}=\operatorname{card}\left(\Omega \cap \operatorname{supp} g^{*}(D)\right) .
$$


Let $\omega$ be a smooth $(1,1)$-form on $X$. We put

$$
A(g, \Omega, \omega)=\int_{\Omega} g^{*} \omega .
$$

Let $B$ be another Riemann surface, and let $\Omega^{\prime}$ be a relatively compact open subset of $B$. Given a proper, surjective holomorphic map $\pi: Y \rightarrow B$, we put

$$
\operatorname{disc}\left(\pi, \Omega^{\prime}\right)=\sum_{x \in \pi^{-1}\left(\Omega^{\prime}\right)} \operatorname{ord}_{x}(\operatorname{ram} \pi) .
$$

Here we denote by $\operatorname{ram} \pi$ the ramification divisor of $\pi$, which is a divisor on $Y$.

Now we introduce the notation of Nevanlinna theory. We consider the case $B=\mathbb{C}$; this means that we consider a proper, surjective holomorphic map $\pi: Y \rightarrow \mathbb{C}$ and a holomorphic map $g: Y \rightarrow X$. For $r>0$, we put $\mathbb{C}(r)=\{z \in \mathbb{C} ;|z|<r\}$ and $Y(r)=\pi^{-1}(\mathbb{C}(r))$. We set

and

$$
\begin{aligned}
& \bar{N}(r, g, D)=\frac{1}{\operatorname{deg} \pi} \int_{1}^{r} \frac{\bar{n}(g, D, Y(t))}{t} d t, \\
& T(r, g, \omega)=\frac{1}{\operatorname{deg} \pi} \int_{1}^{r} \frac{A(g, Y(t), \omega)}{t} d t
\end{aligned}
$$

$$
N_{\text {ram } \pi}(r)=\frac{1}{\operatorname{deg} \pi} \int_{1}^{r} \frac{\operatorname{disc}(\pi, \mathbb{C}(t))}{t} d t .
$$

Let $L$ be a line bundle on $X$. Let $\|\cdot\|_{1}$ and $\|\cdot\|_{2}$ be two Hermitian metrics on $L$. Let $\omega_{1}$ and $\omega_{2}$ be the curvature forms of $\|\cdot\|_{1}$ and $\|\cdot\|_{2}$, respectively. Then we have

$$
T\left(r, g, \omega_{1}\right)=T\left(r, g, \omega_{2}\right)+O(1) \quad \text { when } r \rightarrow \infty,
$$

which follows by Jensen's formula (cf. [LC, IV.2.1]). Therefore we define the characteristic function $T(r, g, L)$ by

$$
T(r, g, L)=T\left(r, g, \omega_{1}\right)+O(1),
$$

which is well-defined up to bounded function on $r$.

In this paper, the following Nevanlinna inequality will be used repeatedly (cf. [Y, p. 242]): Given an effective divisor $D \subset X$ and a holomorphic map $g: Y \rightarrow X$ such that $g(Y) \not \subset D$, we have

$$
\bar{N}(r, g, D) \leq T(r, g,[D])+O(1)
$$

where $[D]$ is the associated line bundle for $D$. Note that the left hand side of (2.1.1) is non-negative for $r>1$.

2.2. Preliminary results. We introduce two results from $[\mathrm{Y}]$ without proofs. For this purpose, we need some notations from moduli theory (cf. [K]). For more details, the reader is refered to $[\mathrm{Y}]$.

Given an integer $q \geq 3$, we use the following notation.

$\overline{\mathcal{M}}_{0, q}$ : the moduli space of $q$-pointed stable curves of genus 0 , where $\overline{\mathcal{M}}_{0, q}$ is a smooth projective variety.

$\mathcal{M}_{0, q}$ : the Zariski open subset in $\overline{\mathcal{M}}_{0, q}$ whose points correspond to smooth curves.

$\mathcal{Z}_{q}: \overline{\mathcal{M}}_{0, q} \backslash \mathcal{M}_{0, q}$, where $\mathcal{Z}_{q}$ is a divisor on $\overline{\mathcal{M}}_{0, q}$.

$\overline{\mathcal{U}}_{0, q} \stackrel{\varpi_{q}}{\rightarrow} \overline{\mathcal{M}}_{0, q}$ : the universal curve, where $\overline{\mathcal{U}}_{0, q}$ is a smooth projective variety and $\varpi_{q}$ is a proper flat morphism. 
$\sigma_{1}, \cdots, \sigma_{q}$ : the universal sections of $\varpi_{q}$, where $\sigma_{i}\left(\overline{\mathcal{M}}_{0, q}\right) \cap \sigma_{j}\left(\overline{\mathcal{M}}_{0, q}\right)=\emptyset$ for $i \neq j$.

$\mathcal{D}_{q}$ : the divisor on $\overline{\mathcal{U}}_{0, q}$ determined by $\sum_{i=1}^{q} \sigma_{i}\left(\overline{\mathcal{M}}_{0, q}\right)$.

$\omega_{q}$ : a fixed Kähler form on $\overline{\mathcal{U}}_{0, q}$.

$\eta_{q}$ : a fixed Kähler form on $\overline{\mathcal{M}}_{0, q}$.

$\kappa_{q}$ : the curvature form of a fixed smooth Hermitian metric on $K_{\overline{\mathcal{U}}_{0, q} / \overline{\mathcal{M}}_{0, q}}\left(\mathcal{D}_{q}\right)$.

$\mathcal{J}=\mathcal{J}^{q}:$ the set $\{(i, j, k) ; 1 \leq i<j<k \leq q\}$.

(Given $\alpha=(i, j, k) \in \mathcal{J})$

$\varphi_{\alpha}$ : the contraction map $\overline{\mathcal{U}}_{0, q} \rightarrow \overline{\mathcal{U}}_{0,3} \simeq \mathbb{P}^{1}$ obtained by forgetting all the markings except $i, j, k$.

Now we state the result of [Y, Theorem 4].

Proposition 1. Let $q \geq 3$ be an integer. For all $\varepsilon>0$, there exists a positive constant $C(q, \varepsilon)>0$ with the following property: Let $Y$ and $B$ be Riemann surfaces, and let $\pi: Y \rightarrow$ $B$ be a proper, surjective holomorphic map. Let $R \subset B$ be a relatively compact, connected open subset whose boundary consists of piecewise analytic curves, and put $F=\pi^{-1}(R)$. Consider the following commutative diagram.

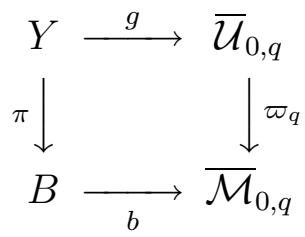

Assume that $b(B) \not \subset \mathcal{Z}_{q}$ and that the meromorphic functions $\varphi_{\alpha} \circ g$ on $Y$ are non-constant for all $\alpha \in \mathcal{J}$. Then we have

$$
\begin{aligned}
A\left(g, F, \kappa_{q}\right) \leq & \bar{n}\left(g, \mathcal{D}_{q}, F\right)+\operatorname{disc}(\pi, R)+\varepsilon A\left(g, F, \omega_{q}\right) \\
& +C(q, \varepsilon)(\operatorname{deg} \pi)\left(A\left(b, R, \eta_{q}\right)+\bar{n}\left(b, \mathcal{Z}_{q}, R\right)+\rho^{+}(R)+\ell\left(g, \partial F, \omega_{q}\right)\right) .
\end{aligned}
$$

Here we denote by $\ell\left(g, \partial F, \omega_{q}\right)$ the length of the $\left.\operatorname{arc} g\right|_{\partial F}: \partial F \rightarrow \overline{\mathcal{U}}_{0, q}$ with respect to the associated Kähler metric of $\omega_{q}$, and we put $\rho^{+}(R)=\max \{0,-($ Euler characteristic of $R)\}$.

Next we state one lemma from $\left[Y\right.$, Lemma 5]. Let $\mathcal{L}$ be the unique line bundle on $\mathbb{P}^{1}$ whose degree is equal to one.

Lemma 1. Let $\alpha \in \mathcal{J}$. Then there exist a line bundle $E_{\alpha}$ on $\overline{\mathcal{M}}_{0, q}$ and a divisor $\Xi_{\alpha}$ on $\overline{\mathcal{U}}_{0, q}$ such that $\varpi_{q}\left(\operatorname{supp} \Xi_{\alpha}\right) \subset \operatorname{supp} \mathcal{Z}_{q}$ and

$$
(q-2) \varphi_{\alpha}^{*} \mathcal{L}=K_{\overline{\mathcal{U}}_{0, q} / \overline{\mathcal{M}}_{0, q}}\left(\mathcal{D}_{q}\right)+\varpi_{q}^{*} E_{\alpha}+\left[\Xi_{\alpha}\right] .
$$

We note that the lemma above is stated only for the case $\alpha=(1,2,3)$ in [Y, Lemma 5]. However the conclusion is obviously valid for all $\alpha \in \mathcal{J}$.

\section{TWO LEMmas FOR THE PROOF OF THEOREM}

Let $V$ and $W$ be smooth projective varieties, and let $\tau: V \rightarrow W$ be a morphism. Let $W_{0}$ be a non-empty Zariski open subset of $W$, and put $V_{0}=\tau^{-1}\left(W_{0}\right)$. Let $L_{1}$ and $L_{2}$ be line bundles on $V$, and let $E$ be an ample line bundle on $W$. Let $Y$ be the same as the theorem, and let $g: Y \rightarrow V$ be a holomorphic map such that $g(Y) \not \subset V \backslash V_{0}$. Under these situations, we have the following two lemmas. 
Lemma 2. Assume that the restrictions $\left.L_{1}\right|_{V_{0}}$ and $\left.L_{2}\right|_{V_{0}}$ are isomorphic on $V_{0}$. Then there is a positive constant $C=C\left(V, W, \tau, W_{0}, L_{1}, L_{2}, E\right)$ which does not depend on $Y$ and $g$ such that

$$
\left|T\left(r, g, L_{1}\right)-T\left(r, g, L_{2}\right)\right| \leq C T(r, \tau \circ g, E)+O(1) .
$$

Lemma 3. Assume that the restriction $\left.L_{1}\right|_{V_{0}}$ is ample on $V_{0}$ and that $L_{1}$ admits a smooth Hermitian metric whose curvature form is semi-positive on $V$. Then there is a positive constant $C=C\left(V, W, \tau, W_{0}, L_{1}, L_{2}, E\right)$ which does not depend on $Y$ and $g$ such that

$$
T\left(r, g, L_{2}\right) \leq C\left(T\left(r, g, L_{1}\right)+T(r, \tau \circ g, E)\right)+O(1) .
$$

Remarks 1. (1) The lemmas above are also true if $V$ is replaced by a finite disjoint union of smooth projective varieties. To generalize Lemma 2 (resp. Lemma 3) to this case, we decompose $V$ into the connected components $V_{1}, \ldots, V_{m}$, and put

$$
C\left(V, W, \tau, W_{0}, L_{1}, L_{2}, E\right)=\max _{1 \leq i \leq m} C\left(V_{i}, W,\left.\tau\right|_{V_{i}}, W_{0},\left.L_{1}\right|_{V_{i}},\left.L_{2}\right|_{V_{i}}, E\right)
$$

where the constant in the right hand side is obtained in Lemma 2 (resp. Lemma 3). For a holomorphic map $g: Y \rightarrow V$ with $g(Y) \not \subset V \backslash V_{0}$, where $V_{0}=\tau^{-1}\left(W_{0}\right)$ as before, we take a connected component $V_{i}$ such that $g(Y) \subset V_{i}$ and apply Lemma 2 (resp. Lemma 3) to the induced map $Y \rightarrow V_{i}$ to deduce our assertion. Note that the characteristic functions $T\left(r, g, L_{1}\right)$ and $T\left(r, g, L_{2}\right)$ are defined in the obvious way for our case.

(2) If $L_{1}$ admits a smooth Hermitian metric whose curvature form is semi-positive, then we have

$$
0 \leq T\left(r, g, L_{1}\right)+O(1)
$$

(3) Assume that there is a morphism $\xi: V \rightarrow V^{\prime}$, where $V^{\prime}$ is a smooth projective variety, and that $L_{1}=\xi^{*} L^{\prime}$ with some ample line bundle $L^{\prime}$ on $V^{\prime}$. Then $L_{1}$ admits a smooth Hermitian metric whose curvature form is semi-positive.

Proof of Lemma 2. Since $\left.L_{1}\right|_{V_{0}}$ and $\left.L_{2}\right|_{V_{0}}$ are isomorphic, there is a divisor $G$ on $V$ such that $[G]=L_{1}-L_{2}$ and $\operatorname{supp} G \subset V \backslash V_{0}$. Since $E$ is ample, we may take linearly equivalent divisors $H_{1}, \ldots, H_{m}$ on $W$ such that:

- $\left[H_{1}\right]=\cdots=\left[H_{m}\right]=n E$ for some positive integer $n$,

- $\operatorname{supp} H_{1} \cap \cdots \cap \operatorname{supp} H_{m}=W \backslash W_{0}$,

- $\tau^{*} H_{i}-G$ and $\tau^{*} H_{i}+G$ are effective divisors on $V$ for all $i=1, \ldots, m$.

Since $g(Y) \not \subset V \backslash V_{0}$, we may take some $i$ such that $g(Y) \not \subset \operatorname{supp} \tau^{*} H_{i} \cup \operatorname{supp} G$. Hence by the Nevanlinna inequality (cf. (2.1.1)), we have

$$
0 \leq T\left(r, g,\left[\tau^{*} H_{i}-G\right]\right)+O(1)=n T(r, \tau \circ g, E)-T(r, g,[G])+O(1),
$$

and

$$
0 \leq T\left(r, g,\left[\tau^{*} H_{i}+G\right]\right)+O(1)=n T(r, \tau \circ g, E)+T(r, g,[G])+O(1) .
$$

Thus we obtain

$$
\left|T\left(r, g, L_{1}\right)-T\left(r, g, L_{2}\right)\right|=|T(r, g,[G])|+O(1) \leq n T(r, \tau \circ g, E)+O(1) .
$$

This proves our lemma. (Put $C=n$.)

Proof of Lemma 3. Since $\left.L_{1}\right|_{V_{0}}$ is ample, there is a positive integer $n$ such that $\left.n L_{1}\right|_{V_{0}}-$ $\left.L_{2}\right|_{V_{0}}$ is very ample on $V_{0}$. We may take effective divisors $H_{1}, \ldots, H_{m}$ on $V$ such that:

- $\left[\left.H_{1}\right|_{V_{0}}\right]=\cdots=\left[\left.H_{m}\right|_{V_{0}}\right]=\left.n L_{1}\right|_{V_{0}}-\left.L_{2}\right|_{V_{0}}$,

- $\left.\left.\operatorname{supp} H_{1}\right|_{V_{0}} \cap \cdots \cap \operatorname{supp} H_{m}\right|_{V_{0}}=\emptyset$. 
We denote by $C_{i}^{\prime}$ the positive constant

$$
C\left(V, W, \tau, W_{0},\left[H_{i}\right], n L_{1}-L_{2}, E\right)
$$

obtained in Lemma 2. Put $C^{\prime}=\max _{1 \leq i \leq m} C_{i}^{\prime}$, which is a positive constant independent of $Y$ and $g$. Since $g(Y) \not \subset V \backslash V_{0}$, we may take some $i$ such that $g(Y) \not \subset \operatorname{supp} H_{i}$. Applying the Nevanlinna inequality (cf. (2.1.1)) and Lemma 2, we get

$$
0 \leq T\left(r, g,\left[H_{i}\right]\right)+O(1) \leq T\left(r, g, n L_{1}-L_{2}\right)+C^{\prime} T(r, \tau \circ g, E)+O(1) .
$$

Put $C=\max \left\{n, C^{\prime}\right\}$ to conclude the proof. Here we note the estimate (3.0.1).

\section{Proof of the theorem}

The proof divides into four steps (from Caim 1 to Claim 4).

4.1. Step 1. We first prove the theorem in the following special case.

Claim 1. If $(X, M, p, D)=\left(\overline{\mathcal{U}}_{0, q}, \overline{\mathcal{M}}_{0, q}, \varpi_{q}, \mathcal{D}_{q}\right), q \geq 3$, then our theorem is true.

Proof. Note that $Z\left(\overline{\mathcal{U}}_{0, q}, \overline{\mathcal{M}}_{0, q}, \varpi_{q}, \mathcal{D}_{q}\right)=\mathcal{Z}_{q}$. Hence the non-degeneracy condition on $b$ assumed in the theorem reads $b(B) \not \subset \mathcal{Z}_{q}$. Let $L$ (resp. $E$ ) be an ample line bundle on $\overline{\mathcal{U}}_{0, q}$ (resp. $\overline{\mathcal{M}}_{0, q}$ ), and let $\varepsilon>0$. Let $Y, B, \pi, g$ and $b$ be the objects for which we want to prove Claim 1. (We assume the non-degeneracy conditions $b(B) \not \subset \mathcal{Z}_{q}$ and $g(Y) \not \subset \mathcal{D}_{q}$.) We consider the following two cases.

Case 1: The functions $\varphi_{\alpha} \circ g$ are non-constant for all $\alpha \in \mathcal{J}$. In this case, we first decompose $B(r)=\pi_{B}^{-1}(\mathbb{C}(r)), r>0$, into connected components $B_{1}(r), \ldots, B_{u_{r}}(r)$. Then, we apply Proposition 1 to the case $R=B_{i}(r)$ and add over $i=1, \ldots, u_{r}$ to obtain

$$
\begin{aligned}
& A\left(g, Y(r), \kappa_{q}\right) \leq \bar{n}\left(g, \mathcal{D}_{q}, Y(r)\right)+\operatorname{disc}(\pi, B(r))+\varepsilon A\left(g, Y(r), \omega_{q}\right) \\
& \quad+C(q, \varepsilon)(\operatorname{deg} \pi)\left(A\left(b, B(r), \eta_{q}\right)+\bar{n}\left(b, \mathcal{Z}_{q}, B(r)\right)+\sum_{i=1}^{u_{r}} \rho^{+}\left(B_{i}(r)\right)+\ell\left(g, \partial Y(r), \omega_{q}\right)\right) .
\end{aligned}
$$

Here $C(q, \varepsilon)$ is the constant which appears in Proposition 1. After dividing by $r \operatorname{deg} \pi_{Y}$, we integrate the inequality and put

$$
L(r)=\frac{1}{\operatorname{deg} \pi_{Y}} \int_{1}^{r} \frac{\ell\left(g, \partial Y(t), \omega_{q}\right)}{t} d t, \quad J(r)=\frac{1}{\operatorname{deg} \pi_{B}} \int_{1}^{r} \frac{\sum_{i=1}^{u_{t}} \rho^{+}\left(B_{i}(t)\right)}{t} d t .
$$

Then we get

$$
\begin{aligned}
T\left(r, g, \kappa_{q}\right) \leq \bar{N}\left(r, g, \mathcal{D}_{q}\right) & +N_{\operatorname{ram} \pi_{Y}}(r)-N_{\operatorname{ram}_{\pi_{B}}}(r)+\varepsilon T\left(r, g, \omega_{q}\right) \\
& +C(q, \varepsilon)\left(T\left(r, b, \eta_{q}\right)+\bar{N}\left(r, b, \mathcal{Z}_{q}\right)+J(r)+(\operatorname{deg} \pi) L(r)\right)
\end{aligned}
$$

for $r>1$. Here we note that $\operatorname{ram} \pi_{Y}=\pi^{*}\left(\operatorname{ram} \pi_{B}\right)+\operatorname{ram} \pi$. Hence we have

$$
\operatorname{disc}\left(\pi_{Y}, \mathbb{C}(r)\right)=(\operatorname{deg} \pi) \operatorname{disc}\left(\pi_{B}, \mathbb{C}(r)\right)+\operatorname{disc}(\pi, B(r))
$$

and

$$
N_{\operatorname{ram} \pi_{Y}}(r)-N_{\operatorname{ram}_{\pi_{B}}}(r)=\frac{1}{\operatorname{deg} \pi_{Y}} \int_{1}^{r} \frac{\operatorname{disc}(\pi, B(t))}{t} d t .
$$

Now we have

Subclaim: The following inequalities hold:

$$
J(r) \leq N_{\operatorname{ram} \pi_{B}}(r) \quad \text { for } r>1,
$$




$$
L(r)=o\left(T\left(r, g, \omega_{q}\right)\right) \| \quad \text { as } r \rightarrow \infty .
$$

Proof of Subclaim. We first prove (4.1.3). We apply Hurwitz's formula to the proper covering map $\left.\pi_{B}\right|_{B_{i}(r)}: B_{i}(r) \rightarrow \mathbb{C}(r)$ to get

$$
\rho\left(B_{i}(r)\right)=\left(\operatorname{deg}\left(\left.\pi_{B}\right|_{B_{i}(r)}\right)\right) \rho(\mathbb{C}(r))+\operatorname{disc}\left(\left.\pi_{B}\right|_{B_{i}(r)}, \mathbb{C}(r)\right) .
$$

Here we put $\rho\left(B_{i}(r)\right)=-\left[\right.$ Euler characteristic of $\left.B_{i}(r)\right]$, and similarly for $\rho(\mathbb{C}(r))$. Since $\rho(\mathbb{C}(r))=-1$ and $\rho\left(B_{i}(r)\right) \geq-1$, we have

$$
\rho^{+}\left(B_{i}(r)\right) \leq \operatorname{disc}\left(\left.\pi_{B}\right|_{B_{i}(r)}, \mathbb{C}(r)\right) .
$$

Hence we have $\sum_{i=1}^{u_{r}} \rho^{+}\left(B_{i}(r)\right) \leq \operatorname{disc}\left(\pi_{B}, \mathbb{C}(r)\right)$, and (4.1.3).

Next we prove (4.1.4) following the method of $[\mathrm{M}]$. In this proof, we denote the covering map $\pi_{Y}: Y \rightarrow \mathbb{C}$ by $p$ to avoid the confusion with the ratio of the circumference $\pi$. Put $g^{*} \omega_{q}=\frac{\sqrt{-1}}{2} G^{2} d p \wedge d \bar{p}$, where $G$ is a $C^{\infty}$-function on $Y \backslash\left\{z \in Y ; p^{\prime}(z)=0\right\}$ with $G \geq 0$. Then we have

$$
\ell(r):=\ell\left(g, \partial Y(r), \omega_{q}\right)=\int_{\partial Y(r)} G r d \arg p
$$

and

$$
A(r):=A\left(g, Y(r), \omega_{q}\right)=\int_{0}^{r}\left(\int_{\partial Y(t)} G^{2} t d \arg p\right) d t .
$$

Put $\delta=\operatorname{deg} p$. Using the Schwarz inequality, we have the following estimates:

$$
\ell(r)^{2} \leq 2 \delta \pi r \int_{\partial Y(r)} G^{2} r d \arg p=2 \delta \pi r \frac{d}{d r} A(r) .
$$

Again by the Schwarz inequality, we have, for $r>1$,

$$
\begin{aligned}
L(r) & =\frac{1}{\delta} \int_{1}^{r} \ell(t) \frac{d t}{t} \leq \sqrt{\frac{2 \pi}{\delta}} \int_{1}^{r} \frac{\sqrt{t A^{\prime}(t)}}{t} d t \\
& =\sqrt{\frac{2 \pi}{\delta}} \int_{1}^{r} \frac{\sqrt{A^{\prime}(t) A(t)}}{\sqrt{A(t) t}} d t \\
& \leq \sqrt{\frac{2 \pi}{\delta}}\left(\int_{1}^{r} \frac{A^{\prime}(t)}{A(t)} d t\right)^{\frac{1}{2}}\left(\int_{1}^{r} \frac{A(t)}{t} d t\right)^{\frac{1}{2}} \\
& =\sqrt{2 \pi} \sqrt{\log A(r)-\log A(1)} \sqrt{T(r)} .
\end{aligned}
$$

Here we put $T(r)=T\left(r, g, \omega_{q}\right)$. Let $\mathcal{E}$ be a subset of $[2, \infty)$ defined by

$$
\mathcal{E}=\left\{r ; T(r)^{2} \leq T^{\prime}(r)\right\}
$$

Then we have

$$
\int_{\mathcal{E}} d r \leq \int_{2}^{\infty} \frac{T^{\prime}(t)}{T(t)^{2}} d t=\frac{1}{T(2)}<\infty .
$$

Furthermore, for $r>2$ and $r \notin \mathcal{E}$, we have

$$
\log A(r)=\log \left(\delta r T^{\prime}(r)\right) \leq \log \delta+\log r+2 \log T(r) .
$$

First, if $g$ is transcendental, then

$$
\lim _{r \rightarrow \infty} \frac{\log r}{T(r)}=0
$$


Hence, outside the set $\mathcal{E}$ with $\int_{\mathcal{E}} d r<\infty$, we have, by (4.1.5) and (4.1.6),

$$
\lim _{r \rightarrow \infty, r \notin \mathcal{E}} \frac{L(r)}{T(r)}=0 .
$$

Next, if $g$ is not transcendental, then

$$
\lim _{r \rightarrow \infty} A(r)<\infty
$$

Thus we have, by (4.1.5),

$$
\lim _{r \rightarrow \infty} \frac{L(r)}{T(r)}=0
$$

Hence, we have $L(r)=o(T(r)) \|$, which proves our assertion.

We combine (4.1.1) and the subclaim above to get

$$
\begin{aligned}
T\left(r, g, K_{\overline{\mathcal{U}}_{0, q} / \overline{\mathcal{M}}_{0, q}}\left(\mathcal{D}_{q}\right)\right) & \leq \bar{N}\left(r, g, \mathcal{D}_{q}\right)+N_{\mathrm{ram} \pi_{Y}}(r)+2 \varepsilon T\left(r, g, \omega_{q}\right) \\
& +C(q, \varepsilon)\left(T\left(r, b, \eta_{q}\right)+\bar{N}\left(r, b, \mathcal{Z}_{q}\right)+N_{\mathrm{ram}_{\pi_{B}}}(r)\right)+O(1) \| .
\end{aligned}
$$

Since $L$ (resp. $E$ ) is an ample line bundle on $\overline{\mathcal{U}}_{0, q}$ (resp. $\overline{\mathcal{M}}_{0, q}$ ), we have the following estimates:

$$
\begin{aligned}
T\left(r, b, \eta_{q}\right) & \leq Q_{1} T(r, b, E)+O(1), \\
T\left(r, g, \omega_{q}\right) & \leq Q_{2} T(r, g, L)+O(1) \\
\bar{N}\left(r, b, \mathcal{Z}_{q}\right) & \leq Q_{3} T(r, b, E)+O(1) \quad(\text { cf. }(2.1 .1)),
\end{aligned}
$$

where $Q_{1}, Q_{2}$ and $Q_{3}$ are positive constants which depend on $q, L$ and $M$, but do not depend on $Y, B, \pi, g, b$ and $\varepsilon$. Hence combining the estimates above with (4.1.7), and replacing $\varepsilon$ with $\varepsilon / 2 Q_{2}$, we obtain the estimate

$$
\begin{aligned}
T\left(r, g, K_{\overline{\mathcal{U}}_{0, q} / \overline{\mathcal{M}}_{0, q}}\left(\mathcal{D}_{q}\right)\right) \leq \bar{N}\left(r, g, \mathcal{D}_{q}\right)+ & N_{\mathrm{ram} \pi_{Y}}(r)+\varepsilon T(r, g, L) \\
& +Q\left(T(r, b, E)+N_{\mathrm{ram}_{\pi_{B}}}(r)\right)+O(1) \|,
\end{aligned}
$$

where $Q=C\left(q, \varepsilon / 2 Q_{2}\right) \max \left\{Q_{1}+Q_{3}, 1\right\}$. Thus we obtain our assertion in the first case that the functions $\varphi_{\alpha} \circ g$ are non-constant for all $\alpha \in \mathcal{J}$.

Case 2: Next we consider the other case, i.e., the function $\varphi_{\alpha} \circ g$ is constant for some $\alpha \in \mathcal{J}$. In this case, we have

$$
T\left(r, \varphi_{\alpha} \circ g, \mathcal{L}\right)=O(1)
$$

By Lemma 1, we have

$$
(q-2) T\left(r, \varphi_{\alpha^{\prime}} \circ g, \mathcal{L}\right)=T\left(r, g, K_{\overline{\mathcal{U}}_{0, q} / \overline{\mathcal{M}}_{0, q}}\left(\mathcal{D}_{q}\right)\right)+T\left(r, b, E_{\alpha^{\prime}}\right)+T\left(r, g,\left[\Xi_{\alpha^{\prime}}\right]\right)+O(1)
$$

for all $\alpha^{\prime} \in \mathcal{J}$, where $E_{\alpha^{\prime}}$ is a line bundle on $\overline{\mathcal{M}}_{0, q}$, and $\Xi_{\alpha^{\prime}}$ is a divisor on $\overline{\mathcal{U}}_{0, q}$ with $\varpi_{q}\left(\operatorname{supp} \Xi_{\alpha^{\prime}}\right) \subset \mathcal{Z}_{q}$. We may take a positive integer $m$ such that $m E+E_{\alpha^{\prime}}$ is an ample line bundle for every $\alpha^{\prime} \in \mathcal{J}$. Then we have

$$
-T\left(r, b, E_{\alpha^{\prime}}\right) \leq m T(r, b, E)+O(1) \text { for all } \alpha^{\prime} \in \mathcal{J} .
$$

We denote by $Q_{\alpha^{\prime}}^{\prime}$ the positive constant

$$
C\left(\overline{\mathcal{U}}_{0, q}, \overline{\mathcal{M}}_{0, q}, \varpi_{q}, \mathcal{M}_{0, q}, \underline{\mathcal{O}}_{\overline{\mathcal{U}}_{0, q}},\left[\Xi_{\alpha^{\prime}}\right], E\right)
$$


obtained in Lemma 2, where $\underline{\mathcal{O}}_{\overline{\mathcal{U}}_{0, q}}$ is the trivial line bundle on $\overline{\mathcal{U}}_{0, q}$. Since $\underline{\mathcal{O}}_{\overline{\mathcal{U}}_{0, q}}$ and $\left[\Xi_{\alpha^{\prime}}\right]$ are isomorphic on $\varpi_{q}^{-1}\left(\mathcal{M}_{0, q}\right)$, and $b(B) \not \subset \mathcal{Z}_{q}$, we may apply Lemma 2 to get

$$
-T\left(r, g,\left[\Xi_{\alpha^{\prime}}\right]\right) \leq Q_{\alpha^{\prime}}^{\prime} T(r, b, E)+O(1) \text { for all } \alpha^{\prime} \in \mathcal{J} \text {. }
$$

Put $Q^{\prime}=m+\max _{\alpha^{\prime} \in \mathcal{J}} Q_{\alpha^{\prime}}^{\prime}$, which is a positive constant independent of $Y, B, \pi, g$ and $b$. Then we have

$$
-T\left(r, b, E_{\alpha^{\prime}}\right)-T\left(r, g,\left[\Xi_{\alpha^{\prime}}\right]\right) \leq Q^{\prime} T(r, b, E)+O(1) \quad \text { for all } \alpha^{\prime} \in \mathcal{J} .
$$

Combining (4.1.9), (4.1.10) and (4.1.11), we conclude the following estimate

$$
T\left(r, g, K_{\overline{\mathcal{U}}_{0, q} / \overline{\mathcal{M}}_{0, q}}\left(\mathcal{D}_{q}\right)\right) \leq Q^{\prime} T(r, b, E)+O(1) .
$$

Now we go back to the original situation of Claim 1 and combine two cases above. Put $C=\max \left\{Q, Q^{\prime}\right\}$. Then $C$ is a positive constant which depends on $q, L, E$ and $\varepsilon$, but does not depend on $Y, B, \pi, g$ and $b$. By (4.1.8) and (4.1.12), we get the estimate (1.0.1), which conclude the proof of Claim 1.

4.2. Step2. We go back to the general situation of the theorem; let $X, M, p$ and $D$ be the same as the theorem. To reduce the proof of the theorem to Claim 1, it is convenient to make the following definition:

Definition 1. A special correspondence $\mathfrak{C}$ is a commutative diagram:

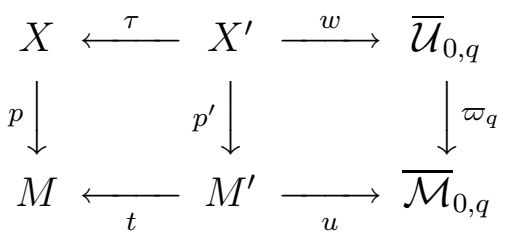

with a smooth projective variety $M^{\prime}$ and a finite disjoint union of smooth projective varieties $X^{\prime}$ provided:

(C1) $\operatorname{dim} M^{\prime}=\operatorname{dim} t\left(M^{\prime}\right)$.

(C2) $t\left(M^{\prime}\right) \not \subset Z(X, M, p, D)$ and $u\left(M^{\prime}\right) \not \subset \mathcal{Z}_{q}$.

(C3) $p^{\prime}\left(X^{\prime \prime}\right)=M^{\prime}$ for every connected component $X^{\prime \prime}$ of $X^{\prime}$.

(C4) There are a non-empty Zariski open subset $U \subset M^{\prime}$ and an effective divisor $G$ of $X^{\prime}$ such that:

(C4a) The restriction $\left.p^{\prime}\right|_{U}:\left(p^{\prime}\right)^{-1}(U) \rightarrow U$ is the base change of $p: X \rightarrow M$ to $U$.

(C4b) $\left.\left(\tau^{*} D+G\right)\right|_{\left(p^{\prime}\right)^{-1}(U)}=\left.\left(w^{*} \mathcal{D}_{q}\right)_{r e d}\right|_{\left(p^{\prime}\right)^{-1}(U)}$.

(C4c) $\left.\left(\tau^{*} K_{X / M}(D)+[G]\right)\right|_{\left(p^{\prime}\right)^{-1}(U)}=\left.w^{*} K_{\overline{\mathcal{U}}_{0, q} / \overline{\mathcal{M}}_{0, q}}\left(\mathcal{D}_{q}\right)\right|_{\left(p^{\prime}\right)^{-1}(U)}$.

Remark 2 . By (C2), (C3) and (C4a), we see that $\tau\left(X^{\prime \prime}\right) \not \subset \operatorname{supp} D$ for every connected component $X^{\prime \prime}$ of $X^{\prime}$, i.e., $\tau^{*} D$ is a divisor on $X^{\prime}$. Hence by (C3) and $(C 4 b), w^{*} \mathcal{D}_{q}$ is also a divisor on $X^{\prime}$.

If a special correspondence $\mathfrak{C}$ exists, then we may "pull-back" the estimate of Claim 1 ; we prove the following.

Claim 2. Let $X, M, p, D, L, E$, and $\varepsilon$ be the same as the theorem. Let $\mathfrak{C}$ be a special correspondence. Then there are a proper Zariski closed subset $S=S(X, M, p, D, \mathfrak{C}) \subset$ $t\left(M^{\prime}\right)$, and a positive constant $C^{\prime}=C^{\prime}(X, M, p, D, L, E, \varepsilon, \mathfrak{C})$ with the following property: 
Let $Y, B$ and $\pi$ be the same as the theorem. Consider the following commutative diagram of holomorphic maps where $g$ is non-constant.

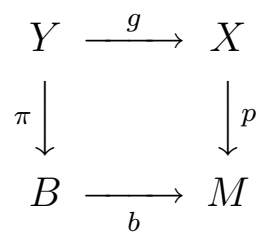

Assume that $b(B) \subset t\left(M^{\prime}\right), b(B) \not \subset S$ and $g(Y) \not \subset \operatorname{supp} D$. Then we have

$$
\begin{aligned}
T\left(r, g, K_{X / M}(D)\right) \leq \bar{N}(r, g, D)+N_{\operatorname{ram} \pi_{Y}} & (r)+\varepsilon T(r, g, L) \\
& +C^{\prime}\left(T(r, b, E)+N_{\operatorname{ram} \pi_{B}}(r)\right)+O(1) \| .
\end{aligned}
$$

Proof. We use the notations in Definition 1. The outline of the proof is as follows: We construct a lifting of (4.2.2):

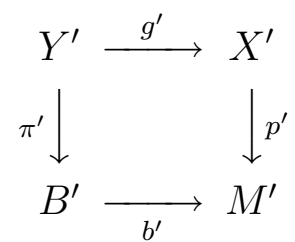

where $Y^{\prime}$ and $B^{\prime}$ are finite ramified covering surfaces of $Y$ and $B$, respectively, and $\pi^{\prime}$ is a proper, surjective holomorphic map. Then we apply Claim 1 to the holomorphic maps $w \circ g^{\prime}: Y^{\prime} \rightarrow \overline{\mathcal{U}}_{0, q}$ and $u \circ b^{\prime}: B^{\prime} \rightarrow \overline{\mathcal{M}}_{0, q}$. Finally we compare the terms $T(r, w \circ$ $\left.g^{\prime}, K_{\overline{\mathcal{U}}_{0, q} / \overline{\mathcal{M}}_{0, q}}\left(\mathcal{D}_{q}\right)\right), \bar{N}\left(r, w \circ g^{\prime}, \mathcal{D}_{q}\right)$, etc., which appear in the resulting estimate, with the terms $T\left(r, g, K_{X / M}(D)\right), \bar{N}(r, g, D)$, etc. to conclude the proof.

In this proof, we denote by

$$
\mathcal{Q}_{1}, \mathcal{Q}_{2}, \mathcal{Q}_{3}, \cdots, \quad \mathcal{Q}_{1}^{\prime}, \mathcal{Q}_{2}^{\prime}, \mathcal{Q}_{3}^{\prime}, \cdots
$$

positive constants which depend on $X, M, p, D, L, E$, and $\mathfrak{C}$, but do not depend on $\varepsilon, Y$, $B, \pi, g$ and $b$.

First we shall find $S$. Replacing $U$ by a smaller non-empty Zariski open subset, we may assume the following conditions on $U$ in addition to $(\mathrm{C} 4 \mathrm{a}),(\mathrm{C} 4 \mathrm{~b})$ and $(\mathrm{C} 4 \mathrm{c})$.

(U1) There is a Zariski open subset $U_{0} \subset t\left(M^{\prime}\right)$ such that $U=t^{-1}\left(U_{0}\right)$, and that the induced map $U \rightarrow U_{0}$ is finite and étale. (Note that the induced map $M^{\prime} \rightarrow t\left(M^{\prime}\right)$ is generically finite by (C1).)

(U2) Let $\Xi$ be an irreducible component of $\left.\left(w^{*} \mathcal{D}_{q}\right)_{\operatorname{red}}\right|_{\left(p^{\prime}\right)^{-1}(U)}$, which is a divisor on $\left(p^{\prime}\right)^{-1}(U)$. Then $\Xi$ is smooth, and the restriction $\left.p^{\prime}\right|_{\Xi}: \Xi \rightarrow U$ is a finite morphism. (Note that the relative dimension of $\left.p^{\prime}\right|_{U}:\left(p^{\prime}\right)^{-1}(U) \rightarrow U$ is equal to one by (C2) and (C4a).)

(U3) $u(U) \subset \mathcal{M}_{0, q} \cdot$ (cf. (C2))

(U4) $M^{\prime} \backslash U$ is a divisor.

Put

$$
S=t\left(M^{\prime}\right) \backslash t(U) .
$$

Then $S \subset t\left(M^{\prime}\right)$ is a proper Zariski closed subset, which depends on $X, M, p, D$ and $\mathfrak{C}$. We note that, by (U1),

$$
t^{-1}(M \backslash S)=U
$$


Now let $Y, B, \pi, g$ and $b$ be the objects in Claim 2 such that $b(B) \subset t\left(M^{\prime}\right)$ and $b(B) \not \subset S$. We shall prove the estimate (4.2.3), where the constant $C^{\prime}$ will be found below.

Lemma 4. There exist liftings $g^{\prime}: Y^{\prime} \rightarrow X^{\prime}$ and $b^{\prime}: B^{\prime} \rightarrow M^{\prime}$ which fit into the following commutative diagram:

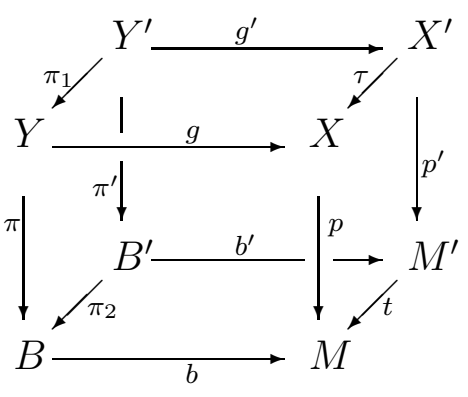

where $\pi_{1}, \pi_{2}$ and $\pi^{\prime}$ are proper, surjective holomorphic maps of Riemann surfaces. Moreover $\pi_{Y^{\prime}}: Y^{\prime} \rightarrow \mathbb{C}$ and $\pi_{B^{\prime}}: B^{\prime} \rightarrow \mathbb{C}$, where $\pi_{Y^{\prime}}=\pi_{Y} \circ \pi_{1}$ and $\pi_{B^{\prime}}=\pi_{B} \circ \pi_{2}$, satisfy the following estimates:

$$
\begin{aligned}
& N_{\mathrm{ram} \pi_{Y^{\prime}}}(r) \leq N_{\operatorname{ram} \pi_{Y}}(r)+\mathcal{Q}_{1} T(r, b, E)+O(1), \\
& N_{\mathrm{ram} \pi_{B^{\prime}}}(r) \leq N_{\operatorname{ram} \pi_{B}}(r)+\mathcal{Q}_{2} T(r, b, E)+O(1) .
\end{aligned}
$$

Proof of Lemma 4. We first construct the lifting $b^{\prime}: B^{\prime} \rightarrow M^{\prime}$. Let $W \subset M$ be the Zariski closure of the image $b(B)$ and let $\mathbb{C}(W)$ be the rational function field of $W$. Let $\mathfrak{K}_{B}$ be the field of all meromorphic functions on $B$. Then we have the injection $\iota: \mathbb{C}(W) \rightarrow \mathfrak{K}_{B}$ of fields, which is naturally defined by $b$. By the assumptions $b(B) \subset t\left(M^{\prime}\right)$ and $b(B) \not \subset S$, we have $W \subset t\left(M^{\prime}\right)$ and $W \not \subset S$. Let $W^{\prime} \subset M^{\prime}$ be an irreducible component of $t^{-1}(W)$. By (4.2.4) and (U1), we observe that the restriction $\left.t\right|_{W^{\prime}}: W^{\prime} \rightarrow W$ is generically finite. Hence the function field $\mathbb{C}\left(W^{\prime}\right)$ is a finite extension of $\mathbb{C}(W)$ with respect to the natural inclusion $\mathbb{C}(W) \subset \mathbb{C}\left(W^{\prime}\right)$ defined by $\left.t\right|_{W^{\prime}}$.

Let $\overline{\mathfrak{K}_{B}}$ be an algebraic closure of $\mathfrak{K}_{B}$. We consider the fields $\mathfrak{K}_{B}, \mathbb{C}(W)$ and $\mathbb{C}\left(W^{\prime}\right)$ as subfields of $\overline{\mathfrak{K}_{B}}$. Then the composite field $F=\mathfrak{K}_{B} \cdot \mathbb{C}\left(W^{\prime}\right)$ is a finite extension of $\mathfrak{K}_{B}$. Hence there exist a Riemann surface $B^{\prime}$ and a proper, surjective holomorphic map $\pi_{2}: B^{\prime} \rightarrow B$ such that the field $\mathfrak{K}_{B^{\prime}}$ is isomorphic to $F$. We also have the holomorphic map $\beta: B^{\prime} \rightarrow W^{\prime}$ where the given inclusion $\mathbb{C}\left(W^{\prime}\right) \subset F$ is induced from this $\beta$. Let $b^{\prime}$ be the composition of $\beta$ and the injection $W^{\prime} \rightarrow M^{\prime}$. Thus we have constructed $B^{\prime}, b^{\prime}$ and $\pi_{2}$. By this construction we have

$$
\operatorname{deg} \pi_{2} \leq \mathcal{Q}_{3},
$$

where we denote by $\mathcal{Q}_{3}$ the degree of the finite map $U \rightarrow U_{0}$ (cf. (U1)).

Next we construct the lifting $g^{\prime}: Y^{\prime} \rightarrow X^{\prime}$. Since the meromorphic function field $\mathfrak{K}_{Y}$ is a finite extension of $\mathfrak{K}_{B}$ with respect to $\pi$, we consider $\mathfrak{K}_{Y}$ as a subfield of $\overline{\mathfrak{K}_{B}}$. Let $F^{\prime}=\mathfrak{K}_{Y} \cdot \mathfrak{K}_{B^{\prime}}$ be the composite field. Then $F^{\prime}$ is a finite extension of $\mathfrak{K}_{Y}$ and $\mathfrak{K}_{B^{\prime}}$. Thus there exist a Riemann surface $Y^{\prime}$, where $\mathfrak{K}_{Y^{\prime}}$ is isomorphic to $F^{\prime}$, and proper, surjective holomorphic maps $\pi_{1}: Y^{\prime} \rightarrow Y$ and $\pi^{\prime}: Y^{\prime} \rightarrow B^{\prime}$. Note that we have a holomorphic map

$$
\left(g \circ \pi_{1}, b^{\prime} \circ \pi^{\prime}\right): Y^{\prime} \rightarrow X \times_{M} M^{\prime} .
$$


Taking into account the facts (C4a), (4.2.4) and $b(B) \not \subset S$, we naturally get the holomorphic map $g^{\prime}: Y^{\prime} \rightarrow X^{\prime}$ from the above map $\left(g \circ \pi_{1}, b^{\prime} \circ \pi^{\prime}\right)$. Thus we have obtained the commutative diagram of the lemma.

We shall prove (4.2.6). By the definitions of $B^{\prime}$ and $b^{\prime}$, the multi-valued morphism $b^{\prime} \circ \pi_{2}^{-1}: B \rightarrow M^{\prime}$ defines the distinct morphism on each branch of $\pi_{2}^{-1}$. Hence by the property (U1) above, we observe that the restriction

$$
\left.\pi_{2}\right|_{\left(b^{\prime}\right)^{-1}(U)}:\left(b^{\prime}\right)^{-1}(U) \rightarrow B
$$

is unramified. Thus we get

$$
\operatorname{supp}\left(\operatorname{ram} \pi_{2}\right) \subset \operatorname{supp}\left(\left(b^{\prime}\right)^{-1}\left(M^{\prime} \backslash U\right)\right),
$$

which yields the estimate

$$
\frac{1}{\operatorname{deg} \pi_{B^{\prime}}} \int_{1}^{r} \frac{\operatorname{disc}\left(\pi_{2}, B(t)\right)}{t} d t \leq\left(\operatorname{deg} \pi_{2}\right) \bar{N}\left(r, b^{\prime}, M^{\prime} \backslash U\right)
$$

for $r>1$ (cf. (U4)). Here we note that $\operatorname{ord}_{x}\left(\operatorname{ram} \pi_{2}\right) \leq \operatorname{deg} \pi_{2}$ for $x \in B^{\prime}$.

Now we shall apply Lemma 2 for the map $t: M^{\prime} \rightarrow M$ to estimate the right hand side of (4.2.8). Note that the two line bundles $\underline{\mathcal{O}}_{M^{\prime}}$ and $\left[M^{\prime} \backslash U\right]$ are isomorphic on $U$, where $\underline{\mathcal{O}}_{M^{\prime}}$ is the trivial line bundle on $M^{\prime}$. Here, by (U4), we note that $M^{\prime} \backslash U$ is a divisor on $M^{\prime}$. Taking into account (4.2.4), we denote by $\mathcal{Q}_{4}$ the positive constant

$$
C\left(M^{\prime}, M, t, M \backslash S, \underline{\mathcal{O}}_{M^{\prime}},\left[M^{\prime} \backslash U\right], E\right)
$$

obtained in Lemma 2. Then we have

$$
\begin{array}{rlrl}
\bar{N}\left(r, b^{\prime}, M^{\prime} \backslash U\right) & \leq T\left(r, b^{\prime},\left[M^{\prime} \backslash U\right]\right)+O(1) & & (\text { cf. }(2.1 .1)) \\
& \leq \mathcal{Q}_{4} T\left(r, t \circ b^{\prime}, E\right)+O(1) & & (\text { cf. Lemma 2) } \\
& =\mathcal{Q}_{4} T(r, b, E)+O(1) .
\end{array}
$$

Hence by (4.2.8) and (4.2.7), we get

$$
\begin{aligned}
\frac{1}{\operatorname{deg} \pi_{B^{\prime}}} \int_{1}^{r} \frac{\operatorname{disc}\left(\pi_{2}, B(t)\right)}{t} d t & \leq\left(\operatorname{deg} \pi_{2}\right) \bar{N}\left(r, b^{\prime}, M^{\prime} \backslash U\right) \\
& =\mathcal{Q}_{3} \mathcal{Q}_{4} T(r, b, E)+O(1)
\end{aligned}
$$

Thus by

$$
N_{\mathrm{ram} \pi_{B^{\prime}}}(r)-N_{\operatorname{ram} \pi_{B}}(r)=\frac{1}{\operatorname{deg} \pi_{B^{\prime}}} \int_{1}^{r} \frac{\operatorname{disc}\left(\pi_{2}, B(t)\right)}{t} d t \quad(\text { cf. (4.1.2)) }
$$

we get $(4.2 .6)$. (Put $\mathcal{Q}_{2}=\mathcal{Q}_{3} \mathcal{Q}_{4}$.)

Next we prove (4.2.5). By the definitions of $Y^{\prime}, \pi^{\prime}$ and $\pi_{1}$, the multi-valued morphism $\pi^{\prime} \circ \pi_{1}^{-1}: Y \rightarrow B^{\prime}$ defines the distinct morphism on each branch of $\pi_{1}^{-1}$. Since $\pi_{2}: B^{\prime} \rightarrow B$ is unramified over $B^{\prime} \backslash \operatorname{supp}\left(\operatorname{ram} \pi_{2}\right)$, we observe that the restriction

$$
\left.\pi_{1}\right|_{\left(\pi^{\prime}\right)^{-1}\left(B^{\prime} \backslash \operatorname{supp}\left(\operatorname{ram} \pi_{2}\right)\right)}:\left(\pi^{\prime}\right)^{-1}\left(B^{\prime} \backslash \operatorname{supp}\left(\operatorname{ram} \pi_{2}\right)\right) \rightarrow Y
$$

is unramified. Thus we get

$$
\operatorname{supp}\left(\operatorname{ram} \pi_{1}\right) \subset\left(\pi^{\prime}\right)^{-1}\left(\operatorname{supp}\left(\operatorname{ram} \pi_{2}\right)\right),
$$


which yields the following estimate for $r>1$ :

$$
\begin{aligned}
\frac{1}{\operatorname{deg} \pi_{Y^{\prime}}} \int_{1}^{r} \frac{\operatorname{disc}\left(\pi_{1}, Y(t)\right)}{t} d t & \leq \frac{\operatorname{deg} \pi_{1}}{\operatorname{deg} \pi_{Y^{\prime}}} \int_{1}^{r} \frac{\#\left\{Y^{\prime}(t) \cap \operatorname{supp}\left(\operatorname{ram} \pi_{1}\right)\right\}}{t} d t \\
& \leq \frac{\operatorname{deg} \pi_{1}}{\operatorname{deg} \pi_{B^{\prime}}} \int_{1}^{r} \frac{\#\left\{B^{\prime}(t) \cap \operatorname{supp}\left(\operatorname{ram} \pi_{2}\right)\right\}}{t} d t \\
& \leq \frac{\operatorname{deg} \pi_{1}}{\operatorname{deg} \pi_{B^{\prime}}} \int_{1}^{r} \frac{\operatorname{disc}\left(\pi_{2}, B(t)\right)}{t} d t
\end{aligned}
$$

Here we note that $\operatorname{ord}_{x}\left(\operatorname{ram} \pi_{1}\right) \leq \operatorname{deg} \pi_{1}$ for $x \in Y^{\prime}$. Hence we get

$$
\begin{aligned}
N_{\operatorname{ram} \pi_{Y^{\prime}}}(r)-N_{\operatorname{ram} \pi_{Y}}(r) & =\frac{1}{\operatorname{deg} \pi_{Y^{\prime}}} \int_{1}^{r} \frac{\operatorname{disc}\left(\pi_{1}, Y(t)\right)}{t} d t \quad(\text { cf. (4.1.2)) } \\
& \leq \frac{\operatorname{deg} \pi_{1}}{\operatorname{deg} \pi_{B^{\prime}}} \int_{1}^{r} \frac{\operatorname{disc}\left(\pi_{2}, B(t)\right)}{t} d t \\
& =\mathcal{Q}_{2}\left(\operatorname{deg} \pi_{1}\right) T(r, b, E)+O(1) \quad(\text { cf. (4.2.9)). }
\end{aligned}
$$

Since by the construction of $\pi_{1}$ and (4.2.7), we have

$$
\operatorname{deg} \pi_{1} \leq \operatorname{deg} \pi_{2} \leq \mathcal{Q}_{3} .
$$

Thus we get (4.2.5), which proves our lemma. (Put $\mathcal{Q}_{1}=\mathcal{Q}_{2} \mathcal{Q}_{3}$.)

Now we go back to the proof of the claim. We consider the following two cases.

Case 1: $w \circ g^{\prime}\left(Y^{\prime}\right) \subset \mathcal{D}_{q}$. In this case, we are going to prove the estimate

$$
T\left(r, g, K_{X / M}(D)\right) \leq \mathcal{Q}_{5} T(r, b, E)+O(1),
$$

where $\mathcal{Q}_{5}$ will be given below. Let $\Xi_{1}, \ldots, \Xi_{k}$ be irreducible components of $\left.\left(w^{*} \mathcal{D}_{q}\right)_{\text {red }}\right|_{\left(p^{\prime}\right)^{-1}(U)}$. For $i=1, \ldots, k$, we denote by $\bar{\Xi}_{i}$ the Zariski closure of $\Xi_{i}$ in $X^{\prime}$, and by $\xi_{i}: \tilde{\Xi}_{i} \rightarrow \overline{\Xi_{i}}$ a desingularization of $\overline{\Xi_{i}}$. By (U2), $\Xi_{i}$ is smooth. Thus by Hironaka's theorem, we may assume that $\xi_{i}^{-1}\left(\Xi_{i}\right)$ is isomorphic to $\Xi_{i}$, where $\Xi_{i}$ is a Zariski open subset of $\Xi_{i}$. Let $\xi_{i}^{\prime}: \tilde{\Xi}_{i} \rightarrow X^{\prime}$ be the composition of $\xi_{i}$ and the closed immersion $\overline{\Xi_{i}} \rightarrow X^{\prime}$.

We look at the morphism $t \circ p^{\prime} \circ \xi_{i}^{\prime}: \tilde{\Xi}_{i} \rightarrow M$. Let $\mathcal{Q}_{i}^{\prime}$ be the positive constant

$$
C\left(\tilde{\Xi}_{i}, M, t \circ p^{\prime} \circ \xi_{i}^{\prime}, M \backslash S,\left(t \circ p^{\prime} \circ \xi_{i}^{\prime}\right)^{*} E,\left(\tau \circ \xi_{i}^{\prime}\right)^{*} K_{X / M}(D), E\right)
$$

obtained in Lemma 3. Here we note the following three facts to ensure the assumption of Lemma 3:

(1) $\left(t \circ p^{\prime} \circ \xi_{i}^{\prime}\right)^{-1}(M \backslash S) \underset{(4.2 .4)}{=}\left(p^{\prime} \circ \xi_{i}^{\prime}\right)^{-1}(U)=\xi_{i}^{-1}\left(\Xi_{i}\right)$.

(2) By (U1) and (U2), the induced morphism $\xi_{i}^{-1}\left(\Xi_{i}\right) \rightarrow M$ is a composition of the finite morphism $\xi_{i}^{-1}\left(\Xi_{i}\right) \rightarrow U_{0}$ and the immersion $U_{0} \rightarrow M$. Thus the restriction of the line bundle $\left(t \circ p^{\prime} \circ \xi_{i}^{\prime}\right)^{*} E$ on $\xi_{i}^{-1}\left(\Xi_{i}\right)$ is ample.

(3) $\left(t \circ p^{\prime} \circ \xi_{i}^{\prime}\right)^{*} E$ admits a smooth Hermitian metric whose curvature form is semi-positive (cf. Remarks $1(3)$ ).

Put $\mathcal{Q}_{5}=2 \max _{1 \leq i \leq k} \mathcal{Q}_{i}^{\prime}$. We note that there is some $i$ such that $g^{\prime}\left(Y^{\prime}\right) \subset \overline{\Xi_{i}}$ and $g^{\prime}\left(Y^{\prime}\right) \not \subset \overline{\Xi_{i}} \backslash \Xi_{i}$. Hence there is a holomorphic map $\tilde{g}^{\prime}: Y^{\prime} \rightarrow \tilde{\Xi}_{i}$ such that $\xi_{i}^{\prime} \circ \tilde{g}^{\prime}=g^{\prime}$. Applying Lemma 3, we get

$$
T\left(r, \tilde{g}^{\prime},\left(\tau \circ \xi_{i}^{\prime}\right)^{*} K_{X / M}(D)\right) \leq \mathcal{Q}_{5} T(r, b, E)+O(1) .
$$

Since $\tau \circ \xi_{i}^{\prime} \circ \tilde{g}^{\prime}=g \circ \pi_{1}$, we get (4.2.10). 
Case 2: $w \circ g^{\prime}\left(Y^{\prime}\right) \not \subset \mathcal{D}_{q}$. In this case, we are going to prove the following estimate

$$
\begin{aligned}
T\left(r, g, K_{X / M}(D)\right) \leq \bar{N}(r, g, D)+N_{\mathrm{ram} \pi_{Y}}(r)+\varepsilon T(r, g, L) & \\
& +C^{\prime \prime \prime}\left(T(r, b, E)+N_{\mathrm{ram}_{\pi_{B}}}(r)\right)+O(1) \|
\end{aligned}
$$

where $C^{\prime \prime \prime}$ is a positive constant given below. Let $L^{\prime}$ and $E^{\prime}$ be ample line bundles on $\overline{\mathcal{U}}_{0, q}$ and $\overline{\mathcal{M}}_{0, q}$, respectively. Let $\varepsilon^{\prime}$ be a positive constant. We denote by $C^{\prime \prime}$ the positive constant

$$
C\left(\overline{\mathcal{U}}_{0, q}, \overline{\mathcal{M}}_{0, q}, \varpi_{q}, \mathcal{D}_{q}, L^{\prime}, M^{\prime}, \varepsilon^{\prime}\right)
$$

obtained in Claim 1. By (U3), we may apply Claim 1 to get

$$
\begin{aligned}
T\left(r, w \circ g^{\prime}, K_{\overline{\mathcal{U}}_{0, q} / \overline{\mathcal{M}}_{0, q}}\left(\mathcal{D}_{q}\right)\right) \leq \bar{N}\left(r, w \circ g^{\prime}, \mathcal{D}_{q}\right)+N_{\mathrm{ram} \pi_{Y^{\prime}}}(r)+\varepsilon^{\prime} T\left(r, w \circ g^{\prime}, L^{\prime}\right) \\
+C^{\prime \prime}\left(T\left(r, u \circ b^{\prime}, E^{\prime}\right)+N_{\mathrm{ram} \pi_{B^{\prime}}}(r)\right)+O(1) \| .
\end{aligned}
$$

Next we shall estimate the terms on (4.2.12).

Subclaim. We have the following estimates:

$T\left(r, g, K_{X / M}(D)\right)+T\left(r, g^{\prime},[G]\right) \leq T\left(r, w \circ g^{\prime}, K_{\overline{\mathcal{U}}_{0, q} / \overline{\mathcal{M}}_{0, q}}\left(\mathcal{D}_{q}\right)\right)+\mathcal{Q}_{6} T(r, b, E)+O(1)$,

$$
\begin{gathered}
\bar{N}\left(r, w \circ g^{\prime}, \mathcal{D}_{q}\right) \leq \bar{N}(r, g, D)+\bar{N}\left(r, g^{\prime}, G\right)+\mathcal{Q}_{7} T(r, b, E)+O(1), \\
T\left(r, w \circ g^{\prime}, L^{\prime}\right) \leq \mathcal{Q}_{8}(T(r, g, L)+T(r, b, E))+O(1), \\
T\left(r, u \circ b^{\prime}, E^{\prime}\right) \leq \mathcal{Q}_{9} T(r, b, E)+O(1) .
\end{gathered}
$$

Proof of (4.2.13). Taking into account (C4c), we denote by $\mathcal{Q}_{6}$ the positive constant

$$
C\left(X^{\prime}, M, t \circ p^{\prime}, M \backslash S, w^{*} K_{\overline{\mathcal{U}}_{0, q} / \overline{\mathcal{M}}_{0, q}}\left(\mathcal{D}_{q}\right), \tau^{*} K_{X / M}(D)+[G], E\right)
$$

obtained in Lemma 2, where we note that $\left(t \circ p^{\prime}\right)^{-1}(M \backslash S)=\left(p^{\prime}\right)^{-1}(U)$ (cf. (4.2.4)). Then by

$$
T\left(r, g^{\prime}, \tau^{*} K_{X / M}(D)\right)=T\left(r, g, K_{X / M}(D)\right)+O(1),
$$

we get our estimate (4.2.13) as a direct consequence of Lemma 2 .

Proof of (4.2.14). Put $F=\operatorname{supp}\left(\left(w^{*} \mathcal{D}_{q}\right)_{\text {red }}-\tau^{*} D-G\right)$, which is an effective divisor on $X^{\prime}$ (cf. Remark 2). Taking into account (C4b), we denote by $\mathcal{Q}_{7}$ the positive constant

$$
C\left(X^{\prime}, M, t \circ p^{\prime}, M \backslash S,[F], \underline{\mathcal{O}}_{X^{\prime}}, E\right)
$$

obtained in Lemma 2, where $\underline{\mathcal{O}}_{X^{\prime}}$ is the trivial line bundle on $X^{\prime}$. Then we have

$$
\begin{aligned}
\bar{N}\left(r, w \circ g^{\prime}, \mathcal{D}_{q}\right)-\bar{N}\left(r, g^{\prime}, \tau^{*} D\right)-\bar{N}\left(r, g^{\prime}, G\right) & \leq \bar{N}\left(r, g^{\prime}, F\right) \\
& \leq T\left(r, g^{\prime},[F]\right)+O(1) \\
& \leq \mathcal{Q}_{7} T(r, b, E)+O(1)
\end{aligned}
$$

Hence by

we get (4.2.14).

$$
\bar{N}\left(r, g^{\prime}, \tau^{*} D\right) \leq \bar{N}(r, g, D) \quad(\text { for } r>1)
$$

Proof of (4.2.15). By (C4a) and (U1), the induced morphism $\left(p^{\prime}\right)^{-1}(U) \rightarrow p^{-1}\left(U_{0}\right)$ is finite and étale. Since the induced morphism $\left(p^{\prime}\right)^{-1}(U) \rightarrow X$ is a composition of this finite 
morphism $\left(p^{\prime}\right)^{-1}(U) \rightarrow p^{-1}\left(U_{0}\right)$ and the immersion $p^{-1}\left(U_{0}\right) \rightarrow X$, the restriction of $\tau^{*} L$ on $\left(p^{\prime}\right)^{-1}(U)$ is an ample line bundle. Let $\mathcal{Q}_{8}$ be the positive constant

$$
C\left(X^{\prime}, M, t \circ p^{\prime}, M \backslash S, \tau^{*} L, w^{*} L^{\prime}, E\right)
$$

obtained in Lemma 3 (cf. Remarks 1 (3)). Then our estimate (4.2.15) is a direct consequence of Lemma 3.

Proof of (4.2.16). Since the induced morphism $U \rightarrow M$ is a composition of the finite morphism $U \rightarrow U_{0}$ (cf. (U1)) and the immersion $U_{0} \rightarrow M$, the restriction of $t^{*} E$ on $U$ is an ample line bundle. Let $\mathcal{Q}_{9}$ be the double of the positive constant

$$
C\left(M^{\prime}, M, t, M \backslash S, t^{*} E, u^{*} E^{\prime}, E\right)
$$

obtained in Lemma 3 (cf. Remarks 1 (3)). Then our estimate (4.2.16) is a direct consequence of Lemma 3. This conclude the proof of the subclaim.

We continue to estimate the terms of (4.2.12). Using (4.2.5), (4.2.6), the subclaim above and the estimate

$$
\bar{N}\left(r, g^{\prime}, G\right) \leq T\left(r, g^{\prime},[G]\right)+O(1) \quad(\text { cf. }(2.1 .1)),
$$

and letting $\varepsilon^{\prime}=\varepsilon / \mathcal{Q}_{8}$, we obtain the desired estimate (4.2.11) where $C^{\prime \prime \prime}$ is the constant

$$
C^{\prime \prime \prime}=\max \left\{C^{\prime \prime}, \varepsilon+\mathcal{Q}_{1}+C^{\prime \prime} \mathcal{Q}_{2}+\mathcal{Q}_{6}+\mathcal{Q}_{7}+C^{\prime \prime} \mathcal{Q}_{9}\right\} .
$$

Now we complete the proof of Claim 2 by combining the two cases above. Put $C^{\prime}=$ $\max \left\{\mathcal{Q}_{5}, C^{\prime \prime \prime}\right\}$, which is a positive constant independent of $Y, B, \pi, g$ and $b$. Then, by (4.2.10) and (4.2.11), we obtain the estimate (4.2.3). This conclude the proof of our claim.

4.3. Step 3. We may construct many special correspondences by the following.

Claim 3. Let $X, M, p$ and $D$ be the same as the theorem. Let $V \subset M$ be an irreducible Zariski closed subset with $V \not \subset Z(X, M, p, D)$. Then there is a special correspondence $\mathfrak{C}$ such that $V=t\left(M^{\prime}\right)$.

Proof. Let $v$ be the generic point of $V$ in the sense of scheme theory. Since $V \not \subset Z$, we have $v \in M \backslash Z$. Put $k=\mathbb{C}(V)$. Let $X_{v}$ be the fiber of $p: X \rightarrow M$ over $v$, and let $D_{v} \subset X_{v}$ be the restriction of $D$. Since $v \in M \backslash Z$, we note that $X_{v}$ is a finite disjoint union of smooth projective curves over $k$ and that $D_{v}$ is a reduced divisor.

Let $\alpha: X_{v} \rightarrow \mathbb{P}_{k}^{1}$ be a finite morphism over $k$. We shall construct a desired special correspondence $\mathfrak{C}$ from $\alpha$ by taking models.

Put $F=\operatorname{ram} \alpha$, which is a divisor on $X_{v}$. Let $H \subset \mathbb{P}_{k}^{1}$ be a reduced divisor such that $\alpha\left(\operatorname{supp}\left(D_{v}+F\right)\right) \subset H$ and $\operatorname{deg} H \geq 3$. Then since $D_{v}$ is reduced, there is an effective divisor $G_{0} \subset X_{v}$ such that

$$
\left(\alpha^{*}(H)\right)_{\mathrm{red}}=D_{v}+G_{0} .
$$

By the ramification formula, we have

$$
K_{X_{v}}\left(D_{v}+G_{0}\right)=\alpha^{*} K_{\mathbb{P}_{k}^{1}}(H) .
$$


Note that there is a finite extension $k^{\prime}$ of $k$ such that $H \otimes_{k} k^{\prime} \subset \mathbb{P}_{k^{\prime}}^{1}$ is a union of $k^{\prime}$-rational points. Then by the moduli-space property, we have the following commutative diagram

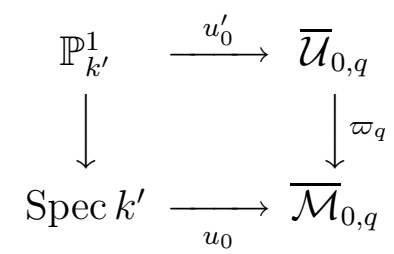

such that the scheme theoretical point $u_{0}\left(\operatorname{Spec} k^{\prime}\right)$ is contained in $\mathcal{M}_{0, q}$, and that:

$$
\left(u_{0}^{\prime}\right)^{*} \mathcal{D}_{q}=H \otimes_{k} k^{\prime}, \quad\left(u_{0}^{\prime}\right)^{*} K_{\overline{\mathcal{U}}_{0, q} / \overline{\mathcal{M}}_{0, q}}\left(\mathcal{D}_{q}\right)=K_{\mathbb{P}_{k}^{1}}(H) \otimes_{k} k^{\prime} .
$$

Put $w_{0}=u_{0}^{\prime} \circ \alpha^{\prime}$, where $\alpha^{\prime}: X_{v} \otimes_{k} k^{\prime} \rightarrow \mathbb{P}_{k^{\prime}}^{1}$ is induced from $\alpha$ by the field extension. Then we get the following commutative diagram:

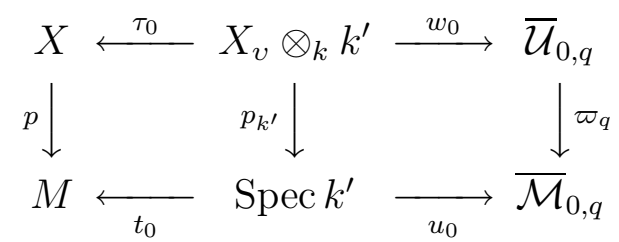

where $t_{0}$ is the natural map induced from $v, p_{k^{\prime}}$ is the base change of $p$ to Spec $k^{\prime}$, and $\tau_{0}$ is the natural map. Using (4.3.1), (4.3.2) and (4.3.3), we have

$$
\left(w_{0}^{*} \mathcal{D}_{q}\right)_{\mathrm{red}}=\left(D_{v}+G_{0}\right) \otimes_{k} k^{\prime}, \quad w_{0}^{*} K_{\overline{\mathcal{U}}_{0, q} / \overline{\mathcal{M}}_{0, q}}\left(\mathcal{D}_{q}\right)=\left(K_{X_{v}}\left(D_{v}+G_{0}\right)\right) \otimes_{k} k^{\prime} .
$$

Now, using Hironaka's theorem, we may take a model of (4.3.4), i.e., a smooth projective variety $M^{\prime}$, a finite disjoint union of smooth projective varieties $X^{\prime}$, and a morphism $p^{\prime}: X^{\prime} \rightarrow M^{\prime}$ such that:

- $\mathbb{C}\left(M^{\prime}\right)=k^{\prime}$.

- The generic fiber of $p^{\prime}$ is isomorphic to $p_{k^{\prime}}: X_{v} \otimes_{k} k^{\prime} \rightarrow \operatorname{Spec} k^{\prime}$.

- $X^{\prime}, M^{\prime}$ and $p^{\prime}$ fit into the commutative diagram (4.2.1) where the morphisms $w, u$, $\tau$ and $t$ are the extensions of $w_{0}, u_{0}, \tau_{0}$ and $t_{0}$, respectively.

- The property (C3) holds.

Then we can easily check that this model of (4.3.4) satisfies the conditions (C1), (C2), (C3) and (C4a). Define a divisor $G$ on $X^{\prime}$ by the Zariski closure of $G_{0} \otimes_{k} k^{\prime}$. Then the conditions (C4b) and (C4c) are consequences of (4.3.5). Thus we have constructed a special correspondence $\mathfrak{C}$ which has the desired property $t\left(M^{\prime}\right)=V$. This proves our claim.

4.4. Step 4. We shall finish the proof of the theorem. Let $X, M, p, D, L, E$ and $\varepsilon$ be the objects in the theorem. For each irreducible Zariski closed subset $V \subset M$ with $V \not \subset Z(X, M, p, D)$, we denote by $\mathfrak{C}_{V}$ the special correspondence constructed in Claim 3, and by $S_{V}$ the proper Zariski closed subset $S\left(X, M, p, D, \mathfrak{C}_{V}\right)$ of $V$ constructed in Claim 2. Let $\mathcal{V}$ be the set of irreducible Zariski closed subsets $V \subset M$ with $V \not \subset Z$. We define the sequence

$$
\mathcal{V}_{1}, \mathcal{V}_{2}, \ldots
$$

of subsets of $\mathcal{V}$ by the following inductive rule. Put $\mathcal{V}_{1}=\{M\}$. Define $\mathcal{V}_{i+1}$ from $\mathcal{V}_{i}$ by

$$
\mathcal{V}_{i+1}=\bigcup_{V^{\prime} \in \mathcal{V}_{i}}\left\{V \in \mathcal{V} ; V \text { is an irreducible component of } S_{V^{\prime}}\right\}
$$


Since the number of the irreducible components of $S_{V}$ is finite for all $V \in \mathcal{V}$, each $\mathcal{V}_{i}$ is a finite set. Since $\operatorname{dim} S_{V}<\operatorname{dim} V$ for all $V \in \mathcal{V}$, we have $\mathcal{V}_{i}=\emptyset$ for $i \geq \operatorname{dim} M+2$. Put

$$
\hat{\mathcal{V}}=\bigcup_{1 \leq i \leq \operatorname{dim} M+1} \mathcal{V}_{i}
$$

Then $\hat{\mathcal{V}}$ is a finite set. Put

$$
C(X, M, p, D, L, E, \varepsilon)=\max _{V \in \hat{\mathcal{V}}} C^{\prime}\left(X, M, p, D, L, E, \varepsilon, \mathfrak{C}_{V}\right),
$$

where the constant $C^{\prime}$ in the right hand side is obtained in Claim 2.

Now for the objects $Y, B, \pi, g$ and $b$ for which we want to prove (1.0.1), we may take a minimal $V \in \hat{\mathcal{V}}$ among the elements in $\hat{\mathcal{V}}$ which have the property $b(B) \subset V$. Then by $b(B) \not \subset Z$, we have $b(B) \not \subset S_{V}$. Hence we may apply Claim 2 to get the estimate (1.0.1). This proves our theorem.

\section{Proof of Corollary 1}

In this section, we prove Corollary 1 . We use the notation of the corollary.

By the Riemann-Roch theorem, we may take a finite map $\pi_{B}: B \rightarrow \mathbb{P}^{1}$ such that

$$
\operatorname{deg} \pi_{B} \leq \operatorname{genus}(B)+1 \quad \text { (cf. [H, IV Ex.1.6]). }
$$

Put $\pi_{Y}=\pi_{B} \circ \pi, B_{0}=\pi_{B}^{-1}(\mathbb{C})$ and $Y_{0}=\pi_{Y}^{-1}(\mathbb{C})$, where we consider $\mathbb{C} \subset \mathbb{P}^{1}$ as a Zariski open subset. Then $B_{0}$ and $Y_{0}$ are Zariski open subsets of $B$ and $Y$, respectively. Put $\pi_{B_{0}}=\left.\pi_{B}\right|_{B_{0}}: B_{0} \rightarrow \mathbb{C}$ and $\pi_{Y_{0}}=\left.\pi_{Y}\right|_{Y_{0}}: Y_{0} \rightarrow \mathbb{C}$. Let $b_{0}: B_{0} \rightarrow M$ be the restriction of $b$, and let $g_{0}: Y_{0} \rightarrow X$ be the restriction of $g$.

We shall apply Nevanlinna theory for $b_{0}$ and $g_{0}$. By the definitions of the functions $\bar{N}\left(r, g_{0}, D\right), N_{\text {ram }_{\pi_{B_{0}}}}(r)$, etc., we get the following estimates:

$$
\begin{gathered}
\lim _{r \rightarrow \infty} \frac{\bar{N}\left(r, g_{0}, D\right)}{\log r} \leq \frac{\bar{n}(g, D, Y)}{\operatorname{deg} \pi_{Y}}, \\
\lim _{r \rightarrow \infty} \frac{N_{\operatorname{ram}_{\pi_{B}}}(r)}{\log r} \leq \frac{\operatorname{disc}\left(\pi_{B}, \mathbb{P}^{1}\right)}{\operatorname{deg} \pi_{B}}=\frac{2 \operatorname{genus}(B)-2}{\operatorname{deg} \pi_{B}}+2 \quad(\text { cf. [H, 2.4]), } \\
\lim _{r \rightarrow \infty} \frac{N_{\operatorname{ram}_{T_{Y_{0}}}}(r)}{\log r} \leq \frac{\operatorname{disc}\left(\pi_{Y}, \mathbb{P}^{1}\right)}{\operatorname{deg} \pi_{Y}}=\frac{2 \operatorname{genus}(Y)-2}{\operatorname{deg} \pi_{Y}}+2 \quad(\text { cf. [H, 2.4]), } \\
\lim _{r \rightarrow \infty} \frac{T\left(r, g_{0}, K_{X / M}(D)\right)}{\log r}=\frac{\operatorname{deg} g^{*} K_{X / M}(D)}{\operatorname{deg} \pi_{Y}}, \\
\lim _{r \rightarrow \infty} \frac{T\left(r, g_{0}, L\right)}{\log r}=\frac{\operatorname{deg} g^{*} L}{\operatorname{deg} \pi_{Y}}, \\
\lim _{r \rightarrow \infty} \frac{T\left(r, b_{0}, E\right)}{\log r}=\frac{\operatorname{deg} b^{*} E}{\operatorname{deg} \pi_{B}} .
\end{gathered}
$$

Now we apply the theorem to $Y_{0}, B_{0},\left.\pi\right|_{Y_{0}}: Y_{0} \rightarrow B_{0}, g_{0}$ and $b_{0}$. Multiplying the both hand sides of the resulting estimate by $\operatorname{deg} \pi_{Y} / \log r$, taking the limit $r \rightarrow \infty$, and using the estimates above, we get the estimate

$$
\begin{aligned}
\operatorname{deg} g^{*} K_{X / M}(D) \leq \bar{n} & (g, D, Y)+(2 \operatorname{genus}(Y)-2)+2(\operatorname{deg} \pi) \operatorname{deg} \pi_{B} \\
& +\varepsilon \operatorname{deg} g^{*} L+C(\operatorname{deg} \pi)\left(\operatorname{deg} b^{*} E+(2 \operatorname{genus}(B)-2)+2 \operatorname{deg} \pi_{B}\right) .
\end{aligned}
$$


Here $C$ is the positive constant obtained in the theorem. We use (5.0.1) to conclude the proof. (Replace $C$ by $\max \{C, 4 C+2\}$.)

\section{The truncated $q$-SMall Function theorem}

In this section, we observe that our theorem implies the truncated $q$-small function theorem very simply.

Let $\pi: Y \rightarrow \mathbb{C}$ be a proper, surjective holomorphic map and let $f$ be a meromorphic function on $Y$. We denote by $T(r, f)$ the spherical characteristic function of $f$, i.e.,

$$
T(r, f)=\frac{1}{\operatorname{deg} \pi} \int_{1}^{r}\left(\int_{Y(t)} f^{*} \omega_{\mathbb{P}^{1}}\right) \frac{d t}{t},
$$

where

$$
\omega_{\mathbb{P}^{1}}=\frac{1}{\left(1+|w|^{2}\right)^{2}} \frac{\sqrt{-1}}{2 \pi} d w \wedge d \bar{w}
$$

is the Fubini-Study form on the projective line $\mathbb{P}^{1}$. Since $\omega_{\mathbb{P}^{1}}$ is the curvature form of the Fubini-Study metric on the line bundle $\mathcal{L}$, we have

$$
T(r, f)=T(r, f, \mathcal{L})+O(1) .
$$

Here, on the right hand side, we consider the meromorphic function $f$ as a holomorphic map $f: Y \rightarrow \mathbb{P}^{1}$. We also put

$$
\bar{N}(r, f)=\bar{N}(r, f,(\infty)),
$$

which counts the number of poles of $f$ without counting multiplicities.

Corollary 2. Let $Y, B, \pi$ be the same as the theorem. Recall that $\mathfrak{K}_{B}$ is the field of all meromorphic functions on $B$. Let $\Phi(x)=\sum_{i=0}^{q} a_{i} x^{i} \in \mathfrak{K}_{B}[x]$ be a polynomial in one variable with coefficients in $\mathfrak{K}_{B}$. Assume that the equation $\Phi(x)=0$ has no multiple solutions in an algebraic closure of $\mathfrak{K}_{B}$. Let $f$ be a non-constant meromorphic function on $Y$ such that $\Phi \circ f \neq 0$ as elements in $\mathfrak{K}_{Y}$, where $\Phi \circ f$ is a meromorphic function on $Y$ defined by

$$
\Phi \circ f(z)=\sum_{i=0}^{q} a_{i}(\pi(z))(f(z))^{i}
$$

Then for all $\varepsilon>0$, there exists a positive constant $C(\varepsilon)>0$ such that

$$
(q-2-\varepsilon) T(r, f) \leq \bar{N}\left(r, \frac{1}{\Phi \circ f}\right)+N_{\operatorname{ram} \pi_{Y}}(r)+C(\varepsilon)\left(N_{\operatorname{ram} \pi_{B}}(r)+\sum_{i=0}^{q} T\left(r, a_{i}\right)\right)+O(1) \| .
$$

Remark 3 . The proof will show that the constant $C(\varepsilon)$ depends only on $\varepsilon$ and $q$. It is an interesting open problem to find an explicit upper bound for $C(\varepsilon)$.

Proof. Put $P_{q}=\underbrace{\mathbb{P}^{1} \times \cdots \times \mathbb{P}^{1}}_{q \text { factors }}$, and let $\tau_{i}: P_{q} \rightarrow \mathbb{P}^{1}$ be the $i$-th projection. Put

$$
L_{q}=\sum_{i=1}^{q} \tau_{i}^{*} \mathcal{L}
$$


which is an ample line bundle on $P_{q}$. Let $x$ be the inhomogeneous coordinate of $\mathbb{P}^{1}=$ $\mathbb{C} \cup\{\infty\}$. We define a rational function $\Psi_{q}$ on $P_{q+2}$ by

$$
\Psi_{q}=\sum_{i=0}^{q}\left(x \circ \tau_{i+1}\right)\left(x \circ \tau_{q+2}\right)^{i} .
$$

We denote by $D_{q}$ the zero locus of $\Psi_{q}$, which is a reduced divisor on $P_{q+2}$. We apply the theorem to the following case:

$$
\begin{gathered}
X=P_{q+2}, M=P_{q+1}, p=\left(\tau_{1}, \ldots, \tau_{q+1}\right), D=D_{q}, \\
L=L_{q+2}, E=L_{q+1}, g=\left(a_{0} \circ \pi, \ldots, a_{q} \circ \pi, f\right), b=\left(a_{0}, \ldots, a_{q}\right) .
\end{gathered}
$$

Note that the condition

$$
b(B) \not \subset Z\left(P_{q+2}, P_{q+1},\left(\tau_{1}, \ldots, \tau_{q+1}\right), D_{q}\right)
$$

is equivalent to the fact that $\Phi(x)$ has no multiple solutions in an algebraic closure of $\mathfrak{K}_{B}$. Then by the theorem, we get

$$
\begin{aligned}
T\left(r, g, K_{X / M}\left(D_{q}\right)\right) \leq \bar{N}\left(r, g, D_{q}\right)+ & N_{\operatorname{ram} \pi_{Y}}(r)+\varepsilon T\left(r, g, L_{q+2}\right) \\
& +C\left(T\left(r, b, L_{q+1}\right)+N_{\operatorname{ram} \pi_{B}}(r)\right)+O(1) \|,
\end{aligned}
$$

where $C$ is the positive constant obtained in the theorem. Put

$$
W=P_{q+2} \backslash\left(\cup_{i=1}^{q+1} \tau_{i}^{-1}(\infty) \cup \tau_{q+1}^{-1}(0)\right) .
$$

Then the restriction of $\Psi_{q}$ on $W$ gives a holomorphic map $\left.\Psi_{q}\right|_{W}: W \rightarrow \mathbb{P}^{1}$. Hence by $\Psi_{q} \circ g=\Phi \circ f$, we conclude

$$
\begin{aligned}
\bar{N}\left(r, g, D_{q}\right) & \leq \bar{N}\left(r, \frac{1}{\Phi \circ f}\right)+\sum_{i=0}^{q} \bar{N}\left(r, a_{i}\right)+\bar{N}\left(r, \frac{1}{a_{q}}\right)+O(1) \\
& \leq \bar{N}\left(r, \frac{1}{\Phi \circ f}\right)+2 \sum_{i=0}^{q} T\left(r, a_{i}\right)+O(1) .
\end{aligned}
$$

Since $\left[D_{q}\right]=q \tau_{q+2}^{*} \mathcal{L}+\sum_{i=1}^{q+1} \tau_{i}^{*} \mathcal{L}$ and $K_{X / M}=-2 \tau_{q+2}^{*} \mathcal{L}$, we have

$$
T\left(r, g, K_{X / M}\left(D_{q}\right)\right)=(q-2) T(r, f)+\sum_{i=0}^{q} T\left(r, a_{i}\right)+O(1) .
$$

We also note the following two estimates:

$$
\begin{gathered}
T\left(r, g, L_{q+2}\right)=T(r, f)+\sum_{i=0}^{q} T\left(r, a_{i}\right)+O(1), \\
T\left(r, b, L_{q+1}\right)=\sum_{i=0}^{q} T\left(r, a_{i}\right)+O(1) .
\end{gathered}
$$


Now by (6.0.2)-(6.0.6), we get

$$
\begin{aligned}
(q-2-\varepsilon) T(r, f) \leq \bar{N}\left(r, \frac{1}{\Phi \circ f}\right) & +N_{\operatorname{ram} \pi_{Y}}(r) \\
& +(2+C+\varepsilon)\left(\sum_{i=0}^{q} T\left(r, a_{i}\right)\right)+C N_{\mathrm{ram} \pi_{B}}(r)+O(1) \| .
\end{aligned}
$$

We replace $C$ by $\max \{C, 2+C+\varepsilon\}$ to conclude the proof.

Corollary 3. Let $a_{1}(z), \ldots, a_{q}(z)$ and $f(z)$ be distinct meromorphic functions on $\mathbb{C}$. Assume that $T\left(r, a_{i}\right)=o(T(r, f)) \|$ as $r \rightarrow \infty$ for $i=1, \ldots, q$. Then we have

$$
(q-1-\varepsilon) T(r, f) \leq \bar{N}(r, f)+\sum_{i=1}^{q} \bar{N}\left(r, \frac{1}{f-a_{i}}\right)+O(1) \|
$$

for all $\varepsilon>0$.

Proof. By post-composing $f, a_{1}, \ldots a_{q}$ with a suitable rotation of $\mathbb{P}^{1}$, we may assume that $a_{i}(z) \not \equiv 0, \infty$ for all $i=1, \ldots, q$. We put $b_{0}(z) \equiv 0, b_{i}(z)=1 / a_{i}(z), i=1, \ldots, q$, and $g(z)=1 / f(z)$. Now we apply Corollary 2 for $\Phi(x)=\left(x-b_{0}(z)\right)\left(x-b_{1}(z)\right) \cdots\left(x-b_{q}(z)\right)$ to get

$$
(q-1-\varepsilon) T(r, g) \leq \bar{N}\left(r, \frac{1}{\Phi \circ g}\right)+o(T(r, g))+O(1) \|
$$

Since

$$
T(r, g)=T(r, f)
$$

and

$$
\bar{N}\left(r, \frac{1}{\Phi \circ g}\right)=\bar{N}(r, f)+\sum_{i=1}^{q} \bar{N}\left(r, \frac{1}{f-a_{i}}\right)+o(T(r, f)) \|,
$$

we get our corollary.

\section{REFERENCES}

[H] R. Hartshorne, Algebraic geometry, Springer-Verlag, Berlin, 1977.

[K] F. Knudsen, Projectivity of the moduli space of stable curves. II, Math. Scand. 52 (1983), 12251265 .

[LC] S. Lang and W. Cherry, Topics in Nevanlinna theory, Lecture Notes in Math. 1433, SpringerVerlag, Heidelberg 1990.

[M] J. Miles, A note on Ahlfors' theory of covering surfaces, Proc. Amer. Math. Soc. 21(1969) 30-32.

[N1] J. Noguchi, Meromorphic mappings of a covering space over $\mathbb{C}^{m}$ into a projective variety and defect relations, Hiroshima Math. J. 6 (1976), 265-280.

[N2] J. Noguchi, Nevanlinna-Cartan theory over function fields and a Diophantine equation, J. Rein Angew. Math. 487 (1997), 61-83.

[NO] J. Noguchi and T. Ochiai, Geometric function theory in several complex variables, Transl. Math. Mon. 80, Amer. Math. Soc., Providence, R.I. 1990.

[O] Ch. Osgood, Sometimes effective Thue-Siegel-Roth-Nevanlinna bounds, or better, J. Number Theory 21 (1985), 347-399.

[S] N. Steinmetz, Eine Varallgemeinerung des zweiten Nevanlinnaschen Hauptsatzes, J. Rein Angew. Math. 368 (1986), 131-141.

[V1] P. Vojta, On algebraic points on curves, Compositio Math. 78 (1991), 29-36.

[V2] P. Vojta, A more general abc conjecture, IMRN (International Mathematics Research Notices) 1998 no $21,1103-1116$. 
$[\mathrm{Y}] \quad$ K. Yamanoi, The second main theorem for small functions and related problems, Acta Math. 192 no.2 (2004), 225-294.

Research Institute for Mathematical Sciences, Kyoto University, Oiwake-cho, SakyoKU, КYOTO, 606-8502, JAPAN

E-mail address: ya@kurims.kyoto-u.ac.jp 